\title{
¿El antecedente de nacimiento pretérmino influye en la gravedad de una infección respiratoria aguda en niños menores de tres años?
}

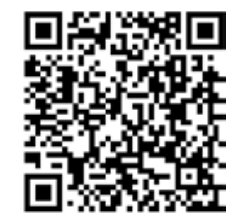

\author{
Gabriela Tijerina-Tijerina, ${ }^{1, *}$ Margarito Marín-Romero, ${ }^{2}$ José Iglesias-Leboreiro, ${ }^{3}$ \\ María Isabel Bernárdez-Zapata, ${ }^{4}$ Mario Enrique Rendón-Macías ${ }^{5}$ \\ ${ }^{1}$ Residente de Pediatría, Facultad Mexicana de Medicina, Universidad La Salle; ${ }^{2}$ División de Pediatría del Hospital Español de \\ México; ${ }^{3}$ División de Pediatría, Unidad de Cuidados Intensivos Neonatales del Hospital Español de México; ${ }^{4}$ Cunero Fisiológico \\ del Hospital Español de México; ${ }^{5}$ Unidad de Investigación en Análisis y Síntesis de la Evidencia, Hospital de Pediatría Centro \\ Médico Nacional Siglo XXI, Instituto Mexicano del Seguro Social, Ciudad de México.
}

\begin{abstract}
RESUMEN
Introducción: Las infecciones respiratorias agudas en niños con antecedente de nacimiento pretérmino podrían ser más graves debido a un retraso en el crecimiento, maduración o daño estructural de sus vías respiratorias. Objetivo: Determinar si el antecedente de ser prematuro influye en el estado de gravedad en niños menores de tres años con infecciones respiratorias agudas. Material y métodos: Estudio transversal, analítico y prospectivo en un servicio de urgencias. Se incluyeron niños $<3$ años atendidos por infecciones respiratorias agudas. Se indagaron las semanas de gestación al nacer y se clasificaron en cuatro grupos (término, pretérmino-tardío [34-36 SDG], pretérminomoderado [28-33 SDG] y pretérmino-extremo [<28 SDG]). La gravedad del episodio de infección respiratoria aguda se evaluó por escala Silverman-Anderson y por requerimiento de hospitalización (sala o terapia intensiva). Resultados: Se incluyeron 295 pacientes, 228 tenían antecedente de nacimiento a término $(77 \%)$ el resto fueron pretérmino, de los cuales, $15 \%$ fue pretérmino tardío $(n=44), 5 \%$ moderado $(n$ $=14$ ) y $3 \%$ pretérmino-extremo $(n=9)$. En general, no hubo diferencias entre los grupos en cuanto a la frecuencia de bronquiolitis y neumonía. La dificultad respiratoria grave se presentó en alrededor de $2 \%$, tanto en los niños de término como en los de pretérmino. Aunque la frecuencia de hospi-
\end{abstract}

\begin{abstract}
Introduction: Acute respiratory infections in children with a history of preterm birth may be more severe due to a delay in growth, maturation, or structural damage to the airways. Objective: To determine whether a history of having been premature influences the condition of severity in children younger than 3 years-old with acute respiratory infections. Material and methods: Cross-sectional, analytical and prospective study in an emergency department. Children < 3 years of age attended by acute respiratory infections were included. Gestation weeks of (WG) at birth were investigated and classified into four groups (term, preterm-late [34-36 WG], preterm-moderate [28-33 WG] and preterm-end [< 28 WG]). The severity of the acute respiratory infection episode was assessed by the Silverman-Anderson scale and the hospitalization requirement (intensive ward or ward). Results: 295 patients were included, 228 had a history of term birth $(77 \%)$, the rest were preterm, of which $15 \%$ were late preterm $(n=44), 5 \%$ moderate preterm $(n=14)$ and $3 \%$ preterm extreme $(n=9)$. In general, there were no differences in the frequency of bronchiolitis and pneumonia between the groups. Severe respiratory distress occurred in about $2 \%$, both in term and preterm infants. Although the frequency of hospitalization and admission to intensive care was slightly higher in the pre-term ones, it was not statistically significant.
\end{abstract}

\footnotetext{
* Correspondencia: GTT, gabriela.tijerinat@gmail.com

Conflicto de intereses: Los autores declaran que no tienen.

Citar como: Tijerina-Tijerina G, Marín-Romero M, Iglesias-Leboreiro J, Bernárdez-Zapata MI, Rendón-Macías ME. ¿El antecedente de nacimiento pretérmino influye en la gravedad de una infección respiratoria aguda en niños menores de tres años? Rev Mex Pediatr. 2019; 86(5):175-180.

[Does having a medical history of pre-term birth influence the severity of an acute respiratory illness in children younger than 3 years?]
} 
talización e ingreso a cuidados intensivos fue un poco mayor en los de pretérmino, no fue estadísticamente significativa. Conclusión: En el presente estudio no se encontró que el antecedente de nacimiento pretérmino tenga influencia sobre el estado de gravedad en niños menores de tres años con infección respiratoria aguda.

Palabras clave: Pretérmino, enfermedades respiratorias, gravedad, urgencias.
Conclusion: In the present study, a history of preterm birth was not found associated with severity in children under three years with acute respiratory infection.

Keywords: Premature, acute respiratory infection, severity medical emergency.

\section{INTRODUCCIÓN}

Las infecciones respiratorias agudas (IRA) son motivo frecuente de consulta en los servicios de urgencias, en especial la bronquiolitis. ${ }^{1}$ La mayoría de las atenciones corresponden a lactantes menores de 12 meses previamente sanos. ${ }^{2}$ Los meses con mayor demanda son diciembre, enero y febrero, principalmente asociados a la circulación del virus sincitial respiratorio (VSR), ${ }^{2}$ y a partir de noviembre por el virus de la influenza. ${ }^{3}$

A pesar que la bronquiolitis es una enfermedad que se autolimita, su comportamiento puede ser grave, en particular entre lactantes con enfermedad pulmonar crónica o cardiopatía congénita. 1,2 Además, se ha demostrado que ser pretérmino (<37 SEG) puede ser otro factor asociado a la gravedad. ${ }^{4}$ En un intento por reducir dicho riesgo en estos pacientes, desde 1998 la Administración de los Alimentos y Drogas (FDA, por sus siglas en inglés) en Estados Unidos de Norteamérica (EUA) autorizó el uso preventivo del anticuerpo monoclonal palivizumab como bloqueador del efecto patogénico del VSR. ${ }^{5}$ Los resultados han sido favorecedores, aunque su uso es limitado por su alto costo.

Desde hace años, los neonatos prematuros tienen mayor probabilidad de sobrevivir en la etapa neonatal, dado por el uso de corticosteroides prenatales, administración de surfactante y mejoras en el apoyo ventilatorio, entre otros. ${ }^{6}$ Estos lactantes, sobre todo los prematuros extremos, nacen durante la fase canalicular y sacular del desarrollo pulmonar, lo cual condiciona mayor riesgo de displasia broncopulmonar (BPD). ${ }^{7}$

La BPD se ha clasificado en "vieja" y "nueva" con base en la edad gestacional al nacimiento, momentos en los que su patogénesis es diferente. En la primera, además del edema alveolar e intersticial, inflamación persistente y fibrosis, se agrega una hipoplasia alveolar, a diferencia de la "nueva", donde la formación alveolar (fase sacular) ya está presente y, por tanto, permite el intercambio gaseoso adecuado. ${ }^{6,8}$ Lo anterior se suma al daño secundario por trauma pulmonar mecánico debido a la ventilación con presión positiva e hiperoxia. Incluso, estos pacientes pueden complicar su condición pulmonar si se agrega reflujo gastroesofágico y neumonía. ${ }^{7}$ Por lo anterior, cuando estos neonatos egresan de Unidades de Cuidados Intensivos Neonatales (UCIN), deben tener cuidados especiales para evitar la progresión del problema respiratorio y enfermedad pulmonar crónica. ${ }^{8,9}$

El objetivo de este estudio fue determinar si el antecedente de ser prematuro influye en el estado de gravedad en niños menores de tres años con IRA.

\section{MATERIAL Y MÉTODOS}

Es un estudio transversal y prolectivo realizado en el servicio de urgencias de un hospital privado de tercer nivel en la Ciudad de México, del 01 de octubre de 2017 al 28 de febrero de 2018.

Se incluyeron niños menores de tres años, quienes acudieron por IRA, con o sin dificultad respiratoria al momento de su atención. Se excluyeron casos con alguna enfermedad crónica.

Todos los pacientes fueron evaluados por los médicos del servicio, quienes recolectaron los datos sobre sus antecedentes, a partir de la información proporcionada por los padres o tutores. En particular se investigó la edad gestacional al nacimiento; en caso de ser $<37$ semanas de gestación (SDG), se agrupó como: pretérminotardío [34-36 SDG], pretérmino-moderado [28-33 SDG] y pretérmino-extremo [<28 SDG]. También se indagó sobre su alimentación al seno materno, esquema de vacunación, enfermedades concomitantes (por ejemplo, reflujo gastroesofágico, cardiopatía, enfermedad pulmonar crónica), uso de medicamentos (incluyendo oxígeno domiciliario) y si acudía a guardería.

Una vez establecido el diagnóstico de IRA, el estado de gravedad fue evaluado por los investigadores a través de la escala de Silverman-Anderson. Los signos vitales y la medición de la oximetría periférica fueron obtenidos por saturómetro de pulso. 
Cabe señalar que la decisión para realizar estudios de laboratorio o de imagenología, así como el manejo de los pacientes, fue por parte de sus médicos tratantes.

El proyecto fue aprobado por el comité de investigación y ética del propio hospital, con número de registro EHE029.

Análisis estadístico. Las variables cualitativas fueron resumidas en frecuencias simples y en porcentaje, mientras que las cuantitativas fueron resumidas como promedio y desviación típica (DT). Los pacientes fueron agrupados de acuerdo a la edad gestacional al momento del nacimiento. Para determinar las diferencias entre los grupos se utilizó la prueba de $\chi^{2}$ en las variables cualitativas y análisis de varianza (ANOVA) para las variables cuantitativas. Se consideró un nivel de significancia estadística con un valor de $\mathrm{p}<0.05$. Los análisis se realizaron con el paquete estadístico SPSS, versión 24.

\section{RESULTADOS}

Se incluyeron 295 niños, 228 tenían antecedente de nacimiento a término (77\%), el resto fueron pretérmino, de los cuales, $15 \%$ fue pretérmino tardío $(\mathrm{n}=44)$, $5 \%$ moderado $(n=14)$ y $3 \%$ pretérmino extremo $(n=9)$.

La comparación de las características entre los grupos de acuerdo a la edad gestacional al nacimiento se describe en la Tabla 1. En los cuatro grupos predominó el sexo masculino, siendo $60 \%$ del total. La

\section{Tabla 1: Características de los pacientes ingresados en urgencias por infección de vías respiratorias según la edad gestacional al nacer.}

\begin{tabular}{|c|c|c|c|c|c|}
\hline & $\begin{array}{l}\text { Término } \\
(\mathrm{N}=228)\end{array}$ & $\begin{array}{l}\text { Pretérmino tardío } \\
\qquad \begin{array}{c}(34-36 \mathrm{SDG}) \\
(\mathrm{N}=44)\end{array}\end{array}$ & $\begin{array}{c}\text { Pretérmino } \\
\text { moderado (29-33 } \\
\text { SDG) }(N=14)\end{array}$ & $\begin{array}{l}\text { Pretérmino extremo } \\
\qquad \begin{array}{c}(<29 \mathrm{SDG}) \\
(\mathrm{N}=9)\end{array}\end{array}$ & \\
\hline Variable & $\mathrm{n}(\%)$ & $\mathrm{n}(\%)$ & $\mathrm{n}(\%)$ & $n(\%)$ & $\mathrm{p}^{*}$ \\
\hline \multicolumn{6}{|l|}{ Sexo } \\
\hline Masculino & $130(61.0)$ & $30(68.2)$ & $8(57.1)$ & $8(88.9)$ & 0.18 \\
\hline Femenino & $89(39.0)$ & $14(31.9)$ & $6(42.9)$ & $1(11.1)$ & \\
\hline $\begin{array}{l}\text { Edad momento evaluación } \\
\text { (meses) promedio ( } \pm 1 D T)\end{array}$ & $14(7.0)$ & $13.3(8.0)$ & $12.7(8.0)$ & $17.1(4.00)$ & $0.56^{\star *}$ \\
\hline Complicaciones periodo neonatal & $4(1.8)$ & $5(15.9)$ & $11(79.6)$ & $9(100.0)$ & $<0.001$ \\
\hline $\begin{array}{l}\text { Asistencia a la ventilación en } \\
\text { la etapa postnatal }\end{array}$ & $3(1.3)$ & $3(6.8)$ & $9(64.3)$ & $9(100.0)$ & $<0.001$ \\
\hline Oxígeno en casa & $1(0.4)$ & 0 & $2(14.3)$ & $1(11.1)$ & $<0.001$ \\
\hline $\begin{array}{l}\text { Hospitalizaciones previas por } \\
\text { infecciones de vías respiratorias }\end{array}$ & 45 (19.7) & $9(20.5)$ & $3(21.4)$ & $3(33.3)$ & 0.43 \\
\hline Retraso psicomotor & $2(0.9)$ & $4(9.1)$ & $8(57.1)$ & $8(88.9)$ & $<0.001$ \\
\hline Lactancia materna $<6$ meses & $98(43.0)$ & $23(52.3)$ & $8(57.1)$ & $7(77.8)$ & 0.018 \\
\hline Reflujo gastroesofágico & $9(3.95)$ & $4(9.1)$ & $2(14.3)$ & $3(33.3)$ & $<0.001$ \\
\hline Cardiopatía congénita & $4(1.8)$ & $2(4.5)$ & $2(14.3)$ & $1(11.1)$ & 0.004 \\
\hline Aplicación palivizumab & $0(0.0)$ & $1(2.3)$ & $1(7.1)$ & $1(11.1)$ & 0.001 \\
\hline \multicolumn{6}{|l|}{ Vacunación contra influenza } \\
\hline No aplicada & $123(53.9)$ & $21(47.7)$ & $5(35.7)$ & $5(55.6)$ & 0.38 \\
\hline No requerida por la edad & $41(18.0)$ & $12(23.9)$ & $4(28.6)$ & 0 & \\
\hline Aplicada & $64(28.1)$ & $11(25.0)$ & $5(35.7)$ & $4(44.4)$ & \\
\hline Acudía a guardería & $102(44.7)$ & $17(38.6)$ & $2(14.3)$ & $4(44.4)$ & 0.13 \\
\hline
\end{tabular}


Tabla 2: Condiciones clínicas al ingreso al servicio de urgencias según el antecedente de pretérmino o término al nacimiento.

\begin{tabular}{|c|c|c|c|c|c|}
\hline & $\begin{array}{l}\text { Término } \\
(\mathrm{N}=228)\end{array}$ & $\begin{array}{l}\text { Pretérmino tardío } \\
\qquad(\mathrm{N}=44)\end{array}$ & $\begin{array}{l}\text { Pretérmino } \\
\text { moderado }(N=14)\end{array}$ & $\begin{array}{c}\text { Pretérmino } \\
\text { extremo }(N=9)\end{array}$ & \\
\hline Variable & $\mathrm{n}(\%)$ & $n(\%)$ & $\mathrm{n}(\%)$ & $\mathrm{n}(\%)$ & $p^{*}$ \\
\hline \multicolumn{6}{|l|}{$\begin{array}{l}\text { Dificultad respiratoria } \\
\text { (Silverman Anderson) }\end{array}$} \\
\hline Sin dificultad $(0)$ & $117(60.0)$ & $20(57.1)$ & $5(38.5)$ & $4(44.4)$ & 0.15 \\
\hline Leve (1-3) & $59(30.3)$ & $10(28.6)$ & $5(38.5)$ & $4(44.4)$ & \\
\hline Moderada (4-6) & $15(7.7)$ & 4 (11.4) & $3(23.1)$ & $1(11.1)$ & \\
\hline Grave $(7-10)$ & $4(2.1)$ & $1(2.9)$ & 0 & 0 & \\
\hline \multicolumn{6}{|l|}{ Signos vitales, promedio $( \pm 1 \mathrm{DT})$} \\
\hline Frecuencia respiratoria & $32.5(8.8)$ & $31.6(7.4)$ & $39.8(14.3)$ & $34.3(11.8)$ & $0.02^{\star *}$ \\
\hline Frecuencia cardiaca & $154(22.0)$ & $154(23.0)$ & $150(25.0)$ & $154(14.0)$ & $0.94^{\star \star}$ \\
\hline TA sistólica (mmHg) & $100(12.5)$ & $103(11.0)$ & $103(12.0)$ & $106(13.0)$ & $0.20^{\star *}$ \\
\hline TA diastólica (mmHg) & $61.2(8.9)$ & $64(9.0)$ & $66(10.0)$ & $63(9.0)$ & $0.06^{\star *}$ \\
\hline Saturación de $\mathrm{O}_{2}$ & $91.7(5.4)$ & $92.3(4.0)$ & $87.5(8.0)$ & $90.5(8.8)$ & $0.04^{\star *}$ \\
\hline Temperatura $\left({ }^{\circ} \mathrm{C}\right)$ & $37.4(0.9)$ & $37.2(0.9)$ & $36.6(0.9)$ & $37.1(0.9)$ & $0.03^{\star *}$ \\
\hline \multicolumn{6}{|l|}{ Diagnóstico clínico } \\
\hline Rinofaringitis & 87 (38.2) & $17(38.6)$ & $3(21.4)$ & $4(44.4)$ & 0.21 \\
\hline Otitis media & $2(0.9)$ & 0 & 0 & 0 & \\
\hline Hiperreactividad bronquial & $38(16.7)$ & $1(2.3)$ & $1(7.1)$ & 0 & \\
\hline Laringotraqueítis & $15(6.6)$ & $10(22.7)$ & $2(14.3)$ & $1(11.1)$ & \\
\hline Bronquiolitis & $64(28.1)$ & $11(25.0)$ & $4(28.6)$ & $2(22.2)$ & \\
\hline Neumonía & $22(9.6)$ & $5(11.4)$ & $4(28.6)$ & $2(22.2)$ & \\
\hline \multicolumn{6}{|l|}{ Prueba viral rápida } \\
\hline Se realizó & $163(71.5)$ & $30(68.2)$ & $9(64.3)$ & $7(77.8)$ & 0.56 \\
\hline Positivo a virus sincicial respiratorio & $41 / 163(25.1)$ & $10(22.7)$ & $4(28.6)$ & $1(11.1)$ & \\
\hline Positivo a influenza $\mathrm{A}$ & $27 / 163(10.6)$ & $4(9.1)$ & $0(0.0)$ & 0 & \\
\hline Positivo a influenza B & $8 / 163(4.9)$ & 0 & $1(7.1)$ & $1(11.1)$ & \\
\hline \multicolumn{6}{|l|}{ Manejo en urgencias } \\
\hline Administración de esteroides & $28(12.3)$ & $6(13.6)$ & $3(21.4)$ & 0 & 0.92 \\
\hline Se tomó gasometría & $17(7.5)$ & $7(15.9)$ & $3(21.4)$ & $2(22.2)$ & 0.01 \\
\hline \multicolumn{6}{|l|}{ Uso de oxígeno/ventilación } \\
\hline Nebulizador templado & $72(31.6)$ & $13(29.5)$ & $8(57.1)$ & $2(22.2)$ & \\
\hline Casco cefálico con oxígeno & $2(0.9)$ & $1(2.3)$ & $1(7.1)$ & 0 & \\
\hline Puntas nasales de alto flujo & $3(1.3)$ & $1(2.3)$ & 0 & 0 & \\
\hline Asistencia a la ventilación & $1(0.4)$ & 0 & 0 & 0 & \\
\hline \multicolumn{6}{|l|}{ Egreso a servicio de urgencias a: } \\
\hline Domicilio & $148(64.9)$ & $27(61.4)$ & $5(35.7)$ & $7(77.8)$ & 0.51 \\
\hline Hospitalización & 75 (32.9) & $15(34.18)$ & $9(64.3)$ & $2(22.2)$ & \\
\hline Unidad de Cuidados Intensivos & $5(2.2)$ & $2(4.5)$ & 0 & 0 & \\
\hline
\end{tabular}

edad al momento de la atención en urgencias no difirió significativamente entre los grupos, teniendo una edad promedio entre 12 y 18 meses de edad. Como era esperado, estadísticamente hubo mayor proporción de pacientes con antecedentes de complicaciones neonatales, así como cardiopatía congénita y requerimiento de asistencia a la ventilación entre menor edad de gestación al nacer. 
En todos los grupos hubo antecedentes de hospitalizaciones previas por problemas respiratorios $(\approx 25 \%)$, sin diferencias entre los grupos.

$\mathrm{Al}$ momento de la atención, hubo cuatro casos con suplementación de oxígeno en su domicilio, tres con antecedente de ser prematuros. Por supuesto, también en este último hubo mayor proporción de reflujo gastroesofágico y retraso psicomotor.

La lactancia materna de al menos seis meses también fue mayor en pacientes de término, aunque sólo en $57 \%$. En los pretérmino, el porcentaje fue mayor en los extremos, seguido de los moderados y leves.

Con respecto a la aplicación de vacunación contra influenza, se aplicó en menos de la mitad de los pacientes. Mientras que palivizumab solamente se otorgó a tres pacientes pretérmino.

\section{Condiciones clínicas al ingreso a urgencias}

En la Tabla 2 se resumen entre los grupos. En general, sólo cinco niños de los 295 estudiados (1.7\%) acudieron con una dificultad respiratoria severa, sin diferencia entre los de pretérmino y de término (2.1 contra $2.9 \%$ ). La mayoría llegó sin dificultad respiratoria, pero resalta la ausencia de condiciones graves en los pretérmino moderados o extremos.

En cuanto a los signos vitales, sólo se encontraron diferencias entre los pretérmino moderados con respecto a los otros grupos. Éstos tuvieron mayor frecuencia respiratoria, menor saturación y temperatura más baja.

Con respecto a la enfermedad respiratoria que motivó la atención, los diagnósticos principales fueron rinofaringitis aguda (37\%), bronquiolitis (27\%) y laringotraqueitis. Neumonía se documentó en 33 pacientes (11.2\%); entre los prematuros ocurrió en $16.4 \%$ (11 pacientes) y en el $9.6 \%$ (22 pacientes) de los nacidos a término $(\mathrm{p}=0.12)$, aunque la frecuencia fue mayor en los prematuros moderados (28\%).

Se realizó prueba rápida para detección de virus respiratorios al $70 \%$ de los pacientes, $30 \%$ fue positivo para el virus sincicial respiratorio, $20 \%$ para influenza (tipo A o B) y el resto negativo (50\%), sin diferencias entre los grupos.

La decisión para la toma de gasometría fue mayor entre los prematuros $(p=0.01)$. La mayoría de los pacientes no requirieron de apoyo ventilatorio, y poco menos de la mitad fueron asistidos con nebulizador templado de oxígeno. Sólo un paciente de término requirió intubación endotraqueal y asistencia ventilatoria.

El manejo hospitalario fue más frecuente para los pretérmino-moderados. Sin embargo, el internamiento en cuidados intensivos sólo se presentó en cinco, en tres de término y en dos de pretérmino. Ningún paciente falleció.

\section{DISCUSIÓN}

Contrario a nuestra hipótesis inicial, los datos de este estudio no apoyan que los niños menores de tres años con IRA tienen mayor gravedad cuando tienen antecedente de haber nacido prematuros. De los cinco pacientes con dificultad respiratoria grave, cuatro fueron de término y sólo uno con el antecedente de haber sido de pretérmino. Lo más probable es que el tamaño de muestra fue el factor más importante para no encontrar esta asociación, dado que hubo pocos pacientes con antecedente de ser pretérmino moderado o extremo.

A nivel mundial, la prematuridad es la segunda causa directa de muerte en niños menores de cinco años, ${ }^{10}$ además de ser un factor de gravedad para la neumonía adquirida en la comunidad. ${ }^{11}$ Sin embargo, estudios previos han demostrado que la mayoría de los niños atendidos en unidades de terapia intensiva son previamente sanos. ${ }^{12}$

La administración de vacunas, en particular la de influenza estacional, ha sido una estrategia comprobada para reducir la incidencia de neumonía comunitaria. ${ }^{3}$ A pesar de su amplia difusión para su aplicación en poblaciones de riesgo, en la que se incluyen a los menores de cinco años de edad y sujetos con alguna morbilidad, a la mitad de nuestros pacientes no se les aplicó. Esta baja tasa de cobertura también ha sido informada en otros estudios. Astrid J y colaboradores encontraron que, a pesar del incremento en años recientes, la cobertura en EUA es menor a $50 \% .{ }^{3} \mathrm{En}$ Hong Kong, J Cowling y su equipo durante el periodo de 2009-2013 encontraron una cobertura de 6.9 a $12.3 \%{ }^{13}$ y en países tropicales y subtropicales ha sido estimada en cinco de cada 1,000 personas, en el año 2016. ${ }^{14}$ Estos datos invitan a insistir en la vacunación de los niños con alto riesgo como los lactantes con antecedente de parto pretérmino.

La aplicación de palivizumab es otra estrategia para reducir la incidencia y gravedad de IRA asociadas al virus sincicial respiratorio. ${ }^{5}$ La principal limitante de su aplicación es su alto costo, quizá por ello la baja administración observada en nuestro estudio; inclusive en países que promueven su aplicación, el complimiento es incompleto. M Perrini y su grupo en EUA entre el año 2009 y 2011 encontraron que sólo $29 \%$ de los niños la recibieron en dosis recomendada. ${ }^{15}$

Otro factor ampliamente estudiado y asociado con la gravedad de las infecciones respiratorias ha sido la edad 
al momento de la neumonía o bronquiolitis. ${ }^{1}$ En un estudio realizado por Mecklin en EUA, los lactantes menores de dos meses de edad eran más propensos a ingresar a la unidad de terapia intensiva y a requerir ventilación mecánica asistida. ${ }^{1}$ Nosotros tuvimos 15 pacientes de esa edad, solamente uno ameritó apoyo ventilatorio.

La principal fortaleza de este estudio fue que la información fue capturada de manera prospectiva, por lo que se pudo obtener información directa de los padres o tutores, y el estado de gravedad se registró de forma precisa y estandarizada. Sin embargo, también reconocemos las limitaciones, una es que no existen criterios claros para hospitalizar pacientes, pero la más importante fue el no disponer de un número mayor de casos graves para conseguir los objetivos.

\section{REFERENCIAS}

1. Mecklin M, Heikkilä P, Korppi M. Low age, low birthweight and congenital heart disease are risk factors for intensive care in infants with bronchiolitis. Acta Paediatr. 2017; 106(12): 2004-2010.

2. Hall CB, Weinberg GA, Blumkin AK, Edwards KM, Staat MA, Schultz AF et al. Respiratory syncytial virus-associated hospitalizations among children less than 24 months of age. Pediatrics. 2013; 132(2): e341-348.

3. Jules A, Grijalva CG, Zhu Y, Talbot HK, Williams JV, Poehling KA et al. Influenza-related hospitalization and ED visits in children less than 5 years: 2000-2011. Pediatrics. 2015; 135(1): e66-74.

4. Figueras-Aloy J, Manzoni P, Paes B, Simões EA, Bont L, Checchia PA et al. Defining the risk and associated morbidity and mortality of severe respiratory syncytial virus infection among preterm infants without chronic lung disease or congenital heart disease. Infect Dis Ther. 2016; 5(4): 417-452.

5. Committee on Infectious Diseases and Bronchiolitis Guidelines Committee. Updated guidance for palivizumab prophylaxis among infants and young children at increased risk of hospitalization for respiratory syncytial virus infection. Pediatrics. 2014; 134(2): 415420.

6. Maduekwe E, DeCristofaro JD. Adjunctive therapies in bronchopulmonary dysplasia. NeoReviews. 2017; 18(3): e173-79.

7. Davidson L, Berkelhamer S. Bronchopulmonary dysplasia: chronic lung disease of infancy and long-term pulmonary outcomes. J Clin Med. 2017; 6(1): 4.

8. Thebaud B. Chronic lung disease in the neonate: past, present, and future. NeoReviews. 2013; 14(5): e252-258.

9. Foglia EE, Kirpalani $H$. Sustained inflation for lung aeration after birth. NeoReviews. 2016; 17(1): e8-17.

10. Blencowe H, Cousens S, Oestergaard MZ, Chou D, Moller A-B, Narwal $R$ et al. National, regional, and worldwide estimates of preterm birth rates in the year 2010 with time trends since 1990 for selected countries: a systematic analysis and implications. Lancet. 2012; 379(9832): 2162-2172.

11. Rudan I. Epidemiology and etiology of childhood pneumonia. Bull World Health Organ. 2008; 86(5): 408-416.

12. Bernhard Resch. Respiratory syncytial virus infection in high-risk infants-an update on palivizumab prophylaxis. Open Microbiol J. 2014; 11(8): 71. doi: 10.2174/1874285801408010071.

13. Cowling BJ, Chan KH, Feng S, Chan EL, Lo JY, Peiris JS et al. The effectiveness of influenza vaccination in preventing hospitalizations in children in Hong Kong, 2009-2013. Vaccine. 2014; 32(41): 5278-5284.

14. Hirve S, Lambach P, Paget J, Vandemaele K, Fitzner J, Zhang $W$. Seasonal influenza vaccine policy, use and effectiveness in the tropics and subtropics-a systematic literature review. Influenza Other Respir Viruses. 2016; 10(4): 254-267.

15. Perrin KM, Begue RE. Use of palivizumab in primary practice. Pediatrics. 2012; 129(1): 55-61. 